\title{
ROBUST CONTROL METHODS A SYSTEMATIC SURVEY
}

\author{
Vojtech Veselý
}

\begin{abstract}
The paper addresses the problem how to recognize a level of robust controller design and is aimed show the difficulties of implementation for practical use. In the first part of paper we introduce the survey of robust controller design for SISO systems with generalization design procedure for structured and unstructured uncertainties. The second part of paper is devoted to MIMO systems. In the frequency domain robust controller design procedure we reduce to independent design of SISO subsystems and in time domain the LMI or BMI approaches with polytopic system description are favorable.
\end{abstract}

K e y w ords: robust control, frequency domain, time domain, polytopic systems, Lyapunov function, linear systems

\section{INTRODUCTION}

During the last decades, robustness has been recognized as a key issue in the analysis and design of control systems. The history of robust control design method based on small-gain-like robustness condition started by developing with the pioneering work of Zames. Only at the end of the 1980's was a practical solution to this problem was found. It is worth to mention some algebraic approaches which followed the seminal works of [4]; [2] and [1]. Theory of robust control system design belongs to those theories which work with a real plant described by a nominal model and model uncertainty. It is a model approval of real plant then there is no gap within the results obtained by control theory and real measurements. Therefore, robust control theory is very fruitful theory to study for practical purposes.

In this paper, we focus our attention in two robust design problems. At first, a problem of robust stabilization of an uncertain system with structured uncertainty when plant is described by the transfer function with linear or multilinear interval for SISO systems and for MIMO systems the polytopic description of systems are considered. In the second, a problem of generalization of robust stabilization of an uncertain system with unstructured uncertainty for SISO and MIMO system is considered for robust decentralized controller design. The last part of paper is devoted to results of three laboratory practice. In this course described basic theory of robust controller design can be consulted also in $[4] ;[2] ;[7] ;[5] ;[8] ;[6] ;[3]$.

\section{ROBUST CONTROLLER DESIGN}

\subsection{Frequency domain. Structured uncertainty}

Consider a closed-loop system comprising the transfer function matrix of the plant $G(s) \in R^{m \times m}$ and the controller $R(s) \in R^{m \times m}$ in the standard feedback configuration, Fig. 1. where $w, u, y, e$ are respectively vectors

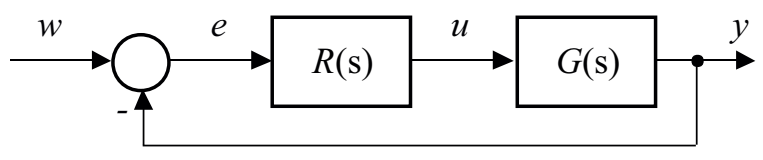

Fig. 1. Standard feedback configuration

of reference, control input, output and control error of compatible dimensions. The problem to addressed in this paper is the design of a robust decentralized controller

$$
R(s)=\operatorname{diag}\left\{R_{i i}(s)\right\}_{m \times m}
$$

that guarantees closed-loop stability and performance over the entire operating range of the controlled plant $G(s)$.

Consider the SISO plant $(m=1)$ with the following type of uncertainties: linear interval, multilinear and nonlinear cases. Let the plant and controller transfer functions is

$$
G(s)=\frac{P_{1}(s)}{P_{2}(s)} \quad R(s)=\frac{R_{1}(s)}{R_{2}(s)}
$$

where $P_{i}(s), i=1,2$ are a linear interval polynomials

$$
P_{i}(s)=p_{o i}+p_{1 i} s+\ldots+p_{n i} s^{n_{i}}
$$

with

$$
p_{j i} \in\left\langle\underline{p_{j i}}, \overline{p_{j i}}\right\rangle \in Q_{i} ; i=1,2 ; j=1,2, \ldots, n_{i} .
$$

The following assumptions about the linear interval polynomials are considered:

- Elements of $p_{i} \in Q_{i}, i=1,2$ are perturbed independently of each other.

- Characteristic polynomials of the plant and the controller are of the same degree.

According to [2] the closed-loop stability problem can be solved using the Generalized Kharitonov Theorem

\footnotetext{
* Institute of Control and Industrial Informatics, Faculty of Electrical Engineering and Information Technnology, Slovak University of Technology, Ilkovivcova 3, 81219 Bratislava, Slovakia, vojtech.vesely@stuba.sk
} 
Theorem 2.1. For a given $R(s)=\left[R_{1}(s) R_{2}(s)\right]$ of real polynomials

- $R(s)$ stabilizes the linear interval polynomials $P(s)=$ $\left[P_{1}(s) P_{2}(s)\right]$ for all $p \in Q$ if and only if the controller stabilizes the extremal transfer function

$$
G_{E}(s)=\left\{\frac{K_{1}(s)}{S_{2}(s)} \bigcup \frac{S_{1}(s)}{K_{2}(s)}\right\} .
$$

- Moreover, if the controller polynomials are of the form

$$
R_{i}(s)=s^{t_{i}}\left(a_{i} s+b_{i}\right)\left(U_{i}(s) Z_{i}(s), i=1,2\right.
$$

then it is sufficient if the controller $R(s)$ stabilizes the Kharitonov transfer function

$$
G_{K}(s)=\frac{K_{1}(s)}{K_{2}(s)}
$$

where $K_{i}(s)=\left\{K_{i}(s)^{1}, K_{i}(s)^{2}, K_{i}(s)^{3}, K_{i}(s)^{4}\right\}$ stand for Kharitonov polynomials corresponding to each $P_{i}(s)$ and

$$
\begin{array}{r}
S_{i}(s)=\left\{\left[K_{i}(s)^{1}, K_{i}(s)^{2}\right],\left[K_{i}(s)^{1}, K_{i}(s)^{3}\right], \ldots\right\} \\
\ldots\left[K_{i}(s)^{2}, K_{i}(s)^{4}\right],\left[K_{i}(s)^{3}, K_{i}(s)^{4}\right]
\end{array}
$$

stand for Kharitonov segments for corresponding $P_{i}(s)$; $U_{i}(s)$ is anti-Hurwitz polynomial; $Z_{i}(s)$ is even or odd polynomial; $a_{i}, b_{i}$ are positive numbers and $t_{i} \geq 0$ is an integer.

Note that

$$
S_{i}(s)^{1}=\lambda K_{i}(s)^{1}+(1-\lambda) K_{i}(s)^{2} ; \lambda \in\langle 0,1\rangle .
$$

Let the plant transfer function of $G(s)$ be written in the following affine form

$$
G(s)=\frac{P_{1}(s)}{P_{2}(s)}=\frac{P_{0,1}(s)+\sum_{i=1}^{p} P_{i, 1}(s) q_{i}}{P_{0,2}(s)+\sum_{i=1}^{p} P_{i, 2}(s) q_{i}}
$$

where $P_{j, 1}(s), P_{j, 2}(s), j=0,1, \ldots, p$ are real polynomials with constant parameters and the uncertainty parameter $q_{i}$ is from the interval

$$
q_{i} \in<\underline{q_{i}}, \overline{q_{i}}>\quad i=1,2, \ldots, p
$$

The description (8) represents a polytope of linear systems, which vertices are

$$
G_{v j}(s)=\frac{P_{v 1, j}(s)}{P_{v 2, j}(s)} \quad j=1,2, \ldots, N ; N=2^{p}
$$

computed for different variable $q_{i}, i=1,2, \ldots, p$ alternatively taken their maximum $\overline{q_{i}}$ and minimum $\underline{q_{i}}$. Based on the Edge theorem the following results can be obtained.
Theorem 2.2. The controller $R(s)=\left[R_{1}(s) R_{2}(s)\right]$ with real polynomials stabilizes the affine system (8) for all $q \in Q$ if and only if the controller stabilizes the following extremal transfer function

$$
G_{P}(s)=\frac{\lambda P_{v 1, i}+(1-\lambda) P_{v 1, j}}{\lambda P_{v 2, i}+(1-\lambda) P_{v 2, j}} .
$$

$i \neq j, i, j=1,2, \ldots, p 2^{p-1} ; \lambda \in<0,1>$ Both $i$ and $j$ have to be taken as the vertices number of corresponding edges. In general the sets of extremal transfer functions (4) and (10) are quite different. While the number of $G_{E}(s)$ equals to 32 , the number of $G_{P}(s)$ depends exponentially on the number of uncertain parameters $q_{i}$.

For the case of multilinear uncertainty let the uncertain plant transfer function be

$$
G(s)=\frac{P_{11}(s) P_{12}(s) \ldots P_{1 n}(s)}{P_{21}(s) P_{22}(s) \ldots P_{2 d}(s)}
$$

where $P_{i j}(s), i=1,2 ; j=1,2, \ldots, n(d)$ belong to the linear interval polynomial. Let $K_{i j}(s)$ and $S_{i j}(s)$ denote the respective Kharitonov polynomials and Kharitonov segments of corresponding $P_{i j}(s)$,respectively. The following theorem holds [2].

Theorem 2.3. The controller $R(s)=\left[R_{1}(s) R_{2}(s)\right]$ stabilizes the multilinear system (11) for the uncertainty box if and only if the polynomials $R(s)$ stabilizes the following Extremal Transfer Function (ETF)

$$
M_{E}(s)=\left\{\frac{S_{11}(s) \ldots S_{1 n}(s)}{K_{21}(s) \ldots K_{2 d}(s)} \bigcup \frac{K_{11}(s) \ldots K_{1 n}(s)}{S_{21}(s) \ldots S_{2 d}(s)}\right\} .
$$

For different type of uncertainty one obtains different number of ETFs. In the laboratory exercises, students can realize robust controller design procedure by two way:

- Using Nejmark D-partition method and calculate the controller parameters for all ETF.

- Design robust controller for the stability worst case of ETF. Check satisfy the stability and performance conditions for all ETFs. If no determine the new worst case for the ETF+ Controller and design new controller For such iterative procedure, we mainly use the BODE diagram approach and phase margin notion.

\subsection{Frequency domain. Unstructured uncer- tainty}

This section deals with the robust controller design for both MIMO and SISO systems using different uncertainty types. Standard feedback configuration for the uncertain system described by additive uncertainty is depicted in Fig. 2. The block diagram in Fig. 2 can easily be trans-

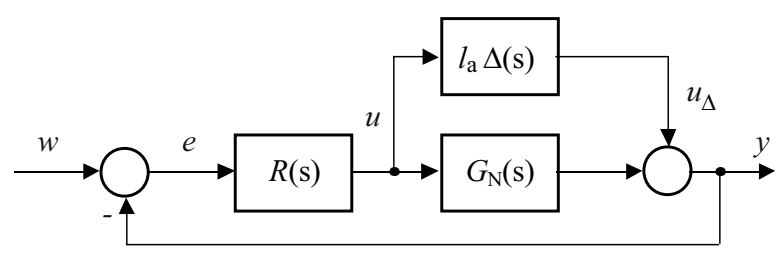

Fig. 2. Standard feedback configuration with additive uncertainty 
formed in the $M-\Delta$ structure [7], where for two (additive and inverse additive) uncertainty types the following results have been obtained

$$
\begin{aligned}
M_{a}(s) & =-\left[I+R(s) G_{N}(s)\right]^{-1} R(s), \\
M_{a i}(s) & =\left[I+G_{N}(s) R(s)\right]^{-1} G_{N}(s) .
\end{aligned}
$$

Robust closed-loop stability conditions are given by following theorem, [7].

Theorem 2.4. Assume that the nominal closed-loop system $M_{k}(s), k=a$, ai is stable and the uncertainties satisfy the following inequality

$$
0<l_{k}(s) \leq l_{k m}(s) \quad k=a, a i .
$$

Then the closed-loop system is stable for all uncertainty models $l_{k}(s), k=a$, ai satisfying (14) if and only if

$$
\sigma_{M}\left(M_{k}(s)\right)<\frac{1}{l_{k}(s)}
$$

where

$$
\begin{aligned}
l_{a}(s) & =\max _{p} \sigma_{M}\left(G_{N}(s)-G_{p}(s)\right) ; p=1,2, \ldots \\
l_{a i}(s) & =\max _{p} \sigma_{M}\left(G_{N}(s)^{-1}-G_{p}(s)^{-1}\right) .
\end{aligned}
$$

Using (13) and (15) after some modifications one obtains

$$
\sigma_{M}\left(M_{T N}\right)<\sigma_{M}\left(\frac{G_{N}}{l_{a}}\right) ; \sigma_{M}\left(S_{N}\right)<\sigma_{M}\left(\frac{G_{N}}{l_{a i}}\right)
$$

where $S_{N}(s), M_{T N}(s)$ are sensitive or complement sensitive functions of closed-loop nominal system, respectively. On the base of Equivalent Subsystem Method (ESM) $[5,8]$ the transfer function of closed-loop nominal system (or sensitivity transfer function) with decentralized controller (1) can transform from stability and performance view to diagonal form with help of equivalent transfer function of nominal model $G_{e}(s)$, ie

$$
M_{T N}\left(G_{N}\right) \simeq M_{T N}\left(G_{e}\right)=\operatorname{diag}\left\{\frac{R_{i} G_{e i}}{1+R_{i} G_{e i}}\right\}_{m \times m}
$$

Due to (17) robust stability conditions (16) reduce to $m$ independent stability conditions:

for additive type uncertainty

$$
\left|\frac{R_{i} G_{e j}}{1+R_{j} G_{e j}}\right|<\left|\frac{G_{N}}{l_{a}}\right|=U_{a}
$$

and for inverse additive type uncertainty

$$
\left|\frac{1}{1+R_{j} G_{e j}}\right|<\left|\frac{G_{N}^{-1}}{l_{a i}}\right|=U_{a i}, \quad j=1,2, \ldots, m .
$$

Robust decentralized controller design procedure consist of the following steps:
- from (18), (19) one can determine the Allowed Frequency Band (AFB) and the maximal value of $M_{T}=$ $\max _{s} U_{a} ;\left(M_{S}=\max _{s} U_{a i}\right)$,

- from the following inequalities

$$
P_{M}>2 \arcsin \left(\frac{1}{2 M_{T}}\right), P_{M}>2 \arcsin \left(\frac{1}{2 M_{S}}\right)
$$

one can determine the phase margin $P_{M}$ and from AFB settling time to design of robust controller,

- for designed controllers the verification of robust stability condition (18), (19) and performance for all subsystems is checked,

- if the stability and performance conditions are meet the design procedure is finished, if no phase margin is increased (settling time is changing),

- for stable plant we use the additive type uncertainty, for unstable inverse additive type one.

\subsection{Robust controller design in time domain: Polytopic system approach}

Consider a linear affine uncertain system

$$
\begin{gathered}
\delta x(t)=A(\alpha) x(t)+B(\alpha) u(t) \\
y(t)=C x(t)
\end{gathered}
$$

where

$$
\begin{aligned}
& \delta x(t)=\dot{x}(t) \text { for continuous-time system, } \\
& \delta x(t)=x(t+1) \text { for discrete-time system. }
\end{aligned}
$$

$x(t) \in R^{n}, u(t) \in R^{m}, y(t) \in R^{l}$ are state, control and output vectors respectively; $A(\alpha), B(\alpha)$ belong to the convex set $S$ with $N$ vertices

$$
\{A(\alpha), B(\alpha)\}=\sum_{i=1}^{N}\left\{A_{i}, B_{i}\right\} \alpha_{i} ; \sum_{i=1}^{N} \alpha_{i}=1, \alpha_{i} \geq 0 .
$$

$A_{i}, B_{i}, C$ are known constant matrices of appropriate dimensions of $i$-th vertex. Such the uncertain system (20), (21) can be equivalently described by a polytopic model given by its vertices

$$
\left\{\left(A_{1}, B_{1}, C\right), \ldots,\left(A_{N}, B_{N}, C\right)\right\}, N=2^{p} .
$$

The feedback control law is considered in the form

$$
u(t)=F C x(t)
$$

where $F$ is a output feedback gain matrix. The uncertain closed-loop polytopic system is then

$$
\delta x(t)=A_{C}(\alpha) x(t)
$$

where

$$
\begin{aligned}
A_{C}(\alpha) & \in\left\{\sum_{i=1}^{N} \alpha_{i} A_{C i}, \sum_{i=1}^{N} \alpha_{i}=1, \alpha_{i} \geq 0\right\}, \\
A_{C i} & =A_{i}+B_{i} F C .
\end{aligned}
$$


To assess the performance quality, a quadratic cost function known from LQ theory is often used. However, in practice the response rate or overshoot are often limited. Therefore we include into the cost function the additional derivative term of state variable to open the possibility to damp the oscillations and limit the response rate. The proposed performance for a continuous-time and for a discrete-time system are given as follows

$$
\begin{gathered}
J_{c}=\int_{0}^{\infty} J(t) d t ; J_{d}=\sum_{t=0}^{\infty} J(t), \\
J(t)=x(t)^{\top} Q x(t)+u(t)^{\top} R u(t)+\delta x(t)^{\top} S \delta x(t)
\end{gathered}
$$

where $Q, S \in R^{n \times n}, R \in R^{m \times m}$ are symmetric positive definite (semidefinite) and definite matrices. The concept of guaranteed cost control is used in a standard way:

DEFINITION 2.1. Let there exist a feedback gain matrix $F$ and a constant $J_{0}\left(x_{0}\right)$ such that

$$
J \leq J_{0}\left(x_{0}\right)
$$

holds for the closed loop system (24). Then the respective control (23) is called the guaranteed cost control and the value of $J_{0}\left(x_{0}\right)$ is the guaranteed cost for the closed-loop system with initial state $x_{0}$.

Let $V(\alpha)$ is Lyapunov function for uncertain closedloop system (24). From LQ theory, see [6] the following lemma for robust stability of system (24) with guaranteed cost holds.

Lemma 2.1. Control algorithm (23) is the guaranteed cost control law for the closed loop system (24) if and only if there exist $V(\alpha)>0$ and constant matrix $F$ such that the following inequality holds

$$
B(\alpha)=\delta V(\alpha)+J(t)<0 .
$$

Moreover, summarizing (integrating) (28) from initial time $t_{0}$ to $t \rightarrow \infty$, the following inequality is obtained

$$
-V\left(t_{0}, \alpha\right)+J_{0}<0
$$

Definition 2.1 with inequality (29) provides guaranteed cost

$$
J_{0}=V\left(t_{0}, \alpha\right)
$$

for closed loop system (24) with control law (23).

The main aim of this part of paper is to show the procedure which we use to design robust PID-PSD controller for laboratory works which stabilizes uncertain polytopic system (20) with guaranteed cost and parameter dependent Lyapunov function defined as

$$
P(\alpha)=\sum_{i=1}^{N} a_{i} P_{i} \text { where } P_{i}=P_{i}^{\top}>0 .
$$

Control algorithm for PID is considered as

$$
u(t)=K_{P} y(t)+K_{I} \int_{0}^{t} y(t) d t+F_{d} \dot{y}_{d}(t) .
$$

The proportional and integral term can be included into the state vector in the common way defining the auxiliary state $z=\int_{0}^{t} y(t)$, ie $\dot{z}(t)=y(t)=C x(t)$ and $\dot{y}_{d}=C_{d} \dot{x}(t)$. The main result on robust PID control stabilization is summarized in the next theorem.

Theorem 2.5. Consider a continuous uncertain linear system (20), (21) with PID controller (31) and cost function (26). The following statements are equivalent:

- Closed loop system (24) is robustly -stable with PDLF

(30) and guaranteed cost with respect to cost function (26):

$$
J \leq J_{0}=x^{\top}(0) P(\alpha) x(0) .
$$

- There exist matrices $P(\alpha)>0$ defined by (30), and $H, G, F$ and $F_{d}$ of the respective dimensions such that

$$
\left[\begin{array}{c}
A_{C i}^{\top} H^{\top}+H A_{C i}+Q+C^{\top} F^{\top} R F C \\
P_{i}-M_{d i}^{\top} H+G^{\top} A_{C i}-M_{d i}^{\top} G-G^{\top} M_{d i}+S
\end{array}\right]<0 .
$$

$A_{C i}=\left(A_{i}+B_{i} F C\right)$ denotes the $i$-th closed loop system vertex, $M_{d i}$ includes the derivative part of the PID controller: $M_{d i}=I-B_{i} F_{d} C_{d}$.

Note, that robust stability condition (32) is in LMI form for stability analysis. For robust PID controller design, where unknown controller parameter matrices $K_{p}, K_{i}, F_{d}$ are to be found, inequality (32) turns to bilinear matrix inequality (BMI).

\section{CASE STUDIES}

In this part of paper, three laboratory works to design of robust controller are described. In the first case, the Magnetic levitation model has been considered. The problem is to design a robust PID controller which will guarantee stability and a desired performance in terms of phase margin over the whole operation range of the plant. The magnetic levitation linearized model is given as follows

$$
G(s)=\frac{y(s)}{u(s)}=\frac{k_{m}}{a s^{2}+b s-1} .
$$

The linear interval model of the magnetic levitation is given as follows $k_{m} \in\langle 2.4,6.8\rangle, a \in\langle 1.34,4.025\rangle \times 10^{-4}$, $b \in\langle 1.7975,5.3895\rangle \times 10^{-6}$. Let the requirement of closedloop performance be given in terms of $M_{T}=1.6, M_{S}=2$ and a phase margin more than $P F M=72$ degree. Using the ETF (4) and the BODE approach the robust PID controller transfer function has been obtained

$$
R(s)=\frac{.02748 s^{2}+1.278 s+8.162}{s}
$$



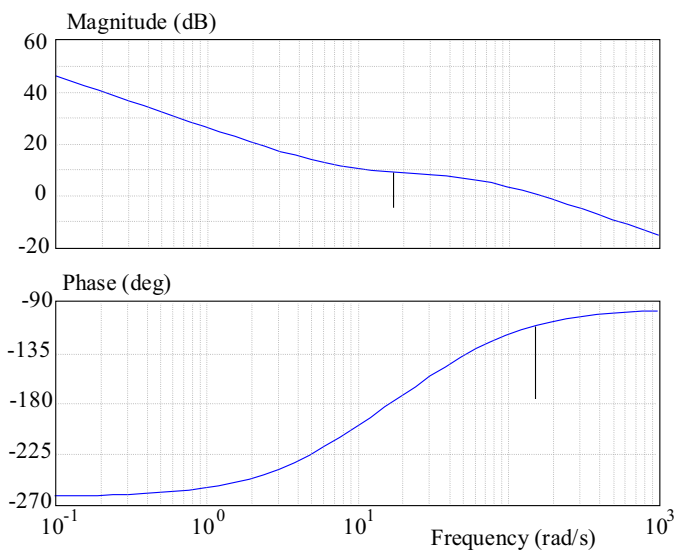

Fig. 3. Bode diagram of open-loop system

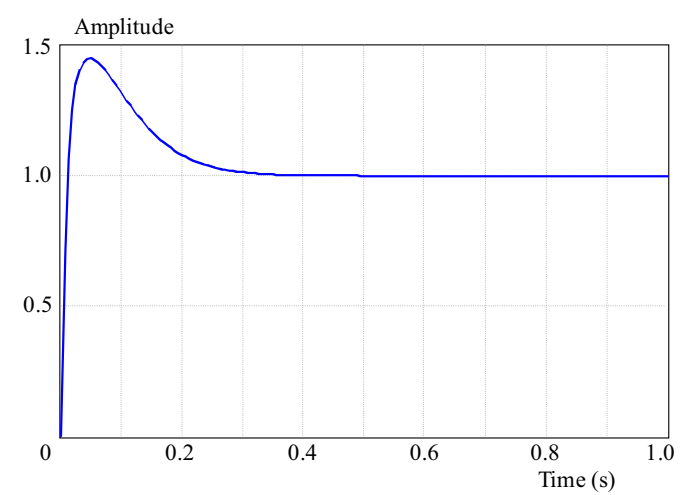

Fig. 5. Closed-loop step response

The Bode diagram of open-loop system for the worst stability case of extremal transfer function is in Fig. 3.

Nyquist diagram with the circle defining the prohibited area for the Nyquist plot is in Fig. 4. Closed loop step response for the worst stability case is given in Fig. 5

The design procedure to design of robust PI controller for Two Tank Proces (TTP) with artificial interaction is illustrated as the second and third group of laboratory exercises for $2 \times 2$ MIMO systems. The TTP has been identified in 3 different working points (position of the valves to flow of water reservoir, level of water). For the first working point the following transfer function is obtained

$$
G_{1}(s)=\left[\begin{array}{cc}
\frac{0.0424 s+.854}{26.93 s^{2}+8.922 s+1} & \frac{0.25}{10 s+1} \\
\frac{0.2}{9 s+1} & \frac{0.0443 s+0.9014}{24.82 s^{2}+7.834 s+1}
\end{array}\right]
$$

Using in this paper described robust controller design procedure for ESM, for settling time $t_{s}=50 \mathrm{~s}$, phase margin $P h=70$ degree and additive type uncertainty the following controllers for the first and second subsystems are obtained:

1. subsystem $P=0.876, I=0.1405, D=0$,

2. subsystem $P=0.8363, I=0.1278, D=0$.

Eigenvalues of closed-loop system for the first working point are Eig $=\{-0.1144 \pm i 0.1949 ;-0.1106 \pm i 0.1662$;

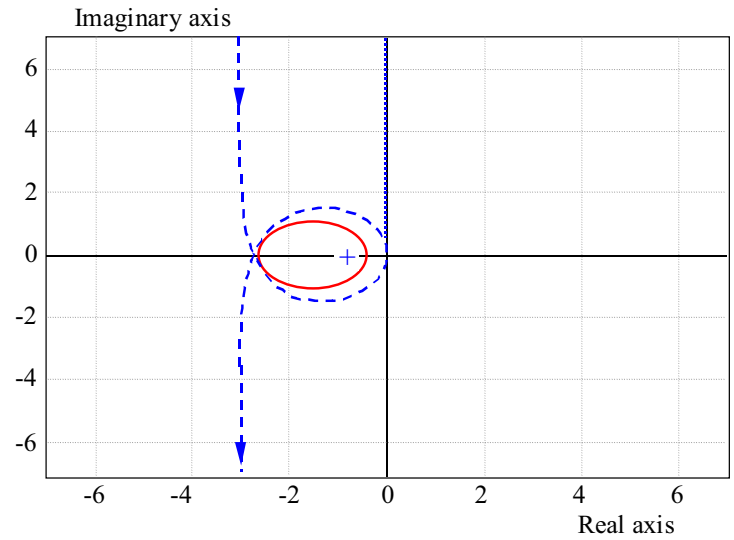

Fig. 4. Nyquist diagram with prohibited area

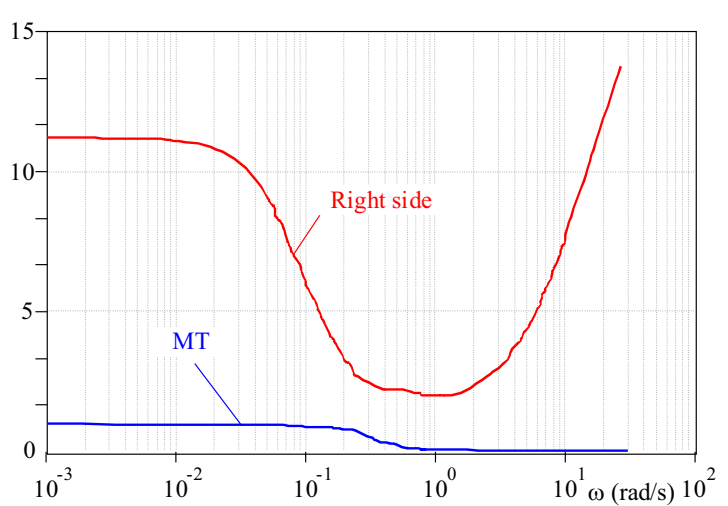

Fig. 6. Robust stability conditions

$-0.1096 \pm i 0.0198 ;-0.0698\}$ Robust stability conditions for additive type uncertainty (18) is given in the Fig. 6 .

Consider above TTP system as a polytopic system in time domain. Robust PI controller can be designed using results of (32). Under the parameters $Q=q I$, $q=0.000001 ; R=r I, r=1 ; S=s I, s=0 ; \varrho=$ $8\left(P_{\max }\right)$, two uncertainties and four vertices one obtain the following PI controller

1. subsystem $P=1.6094 ; I=0.1685$,

2. subsystem $P=0.6661 ; I=0.0723$. Step responses obtained from the real plant for the first and second subsystem is shown in Figs. 7 and 8.

\section{CONCLUSION}

In this paper, a survey of robust control design procedure is given. As presented it is suitable also for the courses given to students in the field of Cybernetics. For SISO system, the course based on design of robust controller for both of interval systems and unstructured uncertainty using small gain theorem. For MIMO systems, our attention to focuses on two directions using polytopic systems with Edge theorem and Ljapunov function and unstructured uncertainty with small gain theorem and mainly recently developed equivalent subsystem method. In the laboratory, every student obtains controller and 


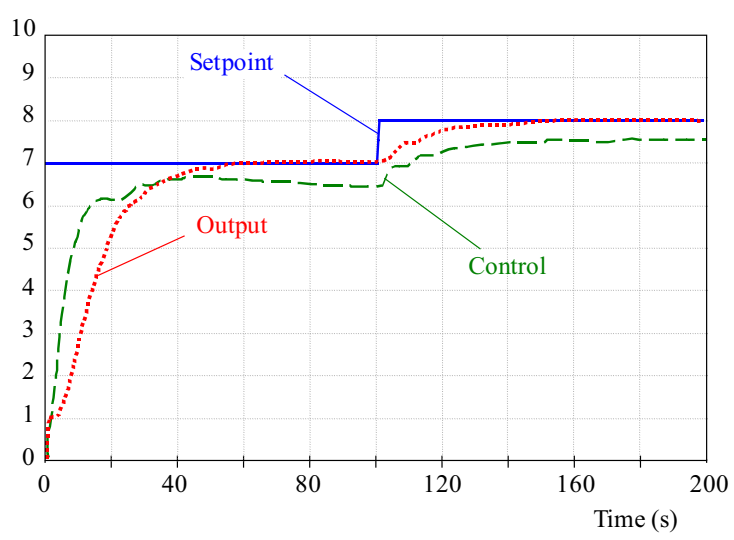

Fig. 7. Real plant step response of subsystem 1

one real plant and a main goal of his exercises is to identify the real plant, create two model uncertainty(polytopic system and additive (multiplicative...) or inverse additive (multiplicative...) uncertainty), design the robust decentralized controller with defined performance and check the obtained results at real plant.

\section{Acknowledgements}

The work has been supported by Grants N $A P V V$ $0211-10$ and 1/1241/12 of the Slovak Scientific Grant Agency.

\section{REFERENCES}

[1] BARLETT, A. C.-HOLlOT, C. V.-LIN, H. : Mathematics of Control, Signals and Systems 1 (1988), 61-71.

[2] BHATTACHARYYA, S. P.-CHAPELLAT, H.-KEEL, L. H. : Robust Control: The Parametric Approach, Prentice Hall, Englewood Cliffs, 1995.

[3] GRMAN, L.-ROSINOVÁ, D.-VESELÝ, V.-KOZÁKOVÁ, A. : Robust Stability Conditions for Polytopic Systems, IJSS 36 No. 15 (Dec 2005), 961-973.

[4] KHARITONOV, V. L. : Asymptotic Stability of an Equilibrium Position of a Family of Systems of Linear Differential Equations, Differential Equations 14 (1979), 1483-1485.

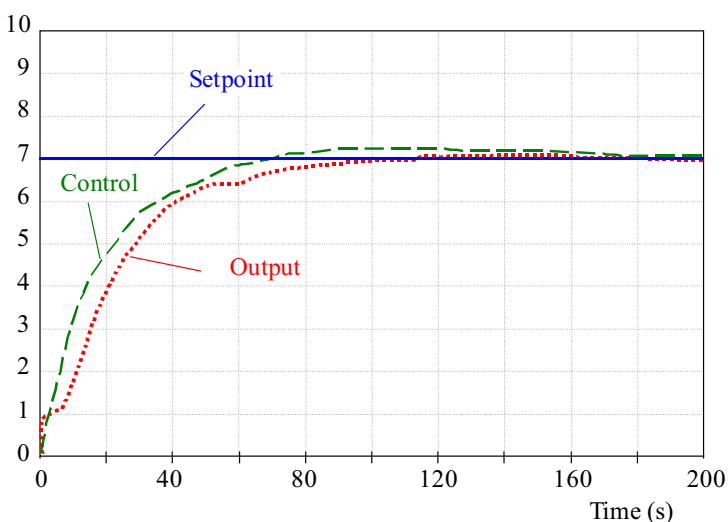

Fig. 8. Real plant step response of subsystem 2

[5] KOZÁKOVÁ, A.-VESELÝ, V.-OSUSKÝ, J. : A New Nyquist-Based Technique for Tuning Robust Decentralezed Controllers, Kybernetika 45 No. 1 (2009), 63-83.

[6] ROSINOVÁ, D.-VESELÝ, V.: Robust PID Decentralized Controller Design using LMI, International Journal of Computers, Communications and Control 2 No. 2 (2007), 195-204.

[7] SKOGESTAD, S.-POSTLETHWAITE, J.: Multivariable Feedback Control: Analysis and Design, John Wiley and Sons yr 1996.

[8] VESELÝ, V.-ROSinOVÁ, D.-KOZÁKOVÁ, A. : Robust Controller Design: New Approaches in the Time and the Frequency Domains, In; Robust Control theory and Applications (Bartoszewicz A., ed.), Intech, 2011, (free online editions www.intechopen.com).

Received 9 March 2012

Vojtech Veselý (Prof, Ing, DrSc) was born in 1940. Since 1964 he has worked at the Department of Automatic Control Systems at the Faculty of Electrical Engineering and Information Technology, Slovak University of Technology in Bratislava, where he has supervised 19 PhD students. Since 1986 he has been Full Professor. His research interests include the areas of power system control, decentralized control of large-scale systems, process control and optimization. He is author and coauthor of more than 250 scientific papers. 